\title{
THE CURRENT-FREE ELECTRIC DOUBLE LAYER IN A CORONAL MAGNETIC FUNNEL
}

\author{
RODERICK W. BOSWELL \\ Space Plasma, Power, and Propulsion Group, Plasma Research Laboratory, Research School of Physical Sciences and Engineering, \\ Australian National University, Canberra, ACT 0200, Australia \\ ECKART MARSCH \\ Max-Planck-Institut für Sonnensystemforschung, 37191 Katlenburg-Lindau, Germany \\ AND \\ Christine Charles \\ Space Plasma, Power, and Propulsion Group, Plasma Research Laboratory, Research School of Physical Sciences and Engineering, \\ Australian National University, Canberra, ACT 0200, Australia \\ Received 2005 June 21; accepted 2006 February 3; published 2006 March 7
}

\begin{abstract}
Current-free double layers (CFDLs) have been recently discovered in a number of laboratory devices, when a low collisional plasma is forced to expand from a high magnetic field source region to a low magnetic field diffusion region. This experimental setup bears a striking resemblance to the natural conditions prevailing in the magnetic funnels of the solar corona. It was commonly thought that magnetic-field-aligned potential disruptions were driven by electron currents, although the theoretical possibility of a CDFL has been known of for some time. Given its recent experimental verification, we make here a contribution to solar plasma physics by investigating the possibility of CFDLs in coronal funnels, which have much in common with the laboratory experiments. Therefore, CFDLs may play an important role in supplying and accelerating plasma in coronal funnels.

Subject headings: acceleration of particles — magnetic fields — plasmas — Sun: corona —

Sun: transition region

Online material: color figure
\end{abstract}

\section{INTRODUCTION}

Double layers (DLs) are spatially isolated, rapid changes of potential in a plasma. Typically, they are quoted as having a width of the order of a Debye length (the correlation length for potential fluctuations in a plasma). DLs have been measured in space and laboratory plasmas. The amplitude of space DLs seems to range from 1 to many $100 \mathrm{eV}$. Recently, current-free double layers (CFDLs) have been discovered in a number of laboratory devices. Based on this experimental evidence, we suggest here that CFDLs may play an important role in supplying and accelerating plasma in coronal funnels.

DLs have been invoked in auroral plasmas (Block 1978) to explain the downward acceleration of kilovolt electrons that collide with the upper atmosphere and produce the visible aurora. Over the past few decades the bulk of the evidence collected by spacecraft and sounding rockets supports the view that at an altitude of about $6000 \mathrm{~km}$ above the aurora, large (Ergun et al. 2001) DLs around $600 \mathrm{~V}$ are associated with upward ion acceleration. Small DLs of the order of the electron temperature have been measured and can provide a sufficient field-aligned potential change to accelerate electrons.

As the Earth's magnetic field is converging down to the aurora, the electrons must be accelerated very nearly parallel to the magnetic field or they will be adiabatically reflected. The latter adds weight to the hypothesis of strictly field-aligned potential structures. Most publications report a field-aligned electron current that is assumed to provide the power for the DL. The origin of the electron current is not clear, but it may be part of a global magnetospheric current system, or possibly of a slow wave bringing energy in from the tail of the magnetosphere (Goertz \& Boswell 1979).

In the solar context, the potential significance of electrostatic DLs in the dissipation of solar flares has been previously realized (see, e.g., the studies of Raadu 1989; Volwerk \& Kuijpers
1994 and references therein). All models of magnetic energy release and particle acceleration by a coronal DL make the assumption that a current is creating the DL. Various currentcircuit geometries in solar flares have been studied (Jacobsen \& Carlqvist 1964; Raadu 1989), with the prediction of a typical electric field in the current sheet of about $450 \mathrm{~V} \mathrm{~m}^{-1}$.

Some early experimental evidence of a DL in a current-sheet configuration has been presented by Stenzel et al. (1983). Most analytical work on DLs has been related to current-driven systems, although Perkins \& Sun (1981) predicted the existence of current-free solutions. The first experimental evidence of a CFDL in a two-electron-population plasma was reported by Hairapetian \& Stenzel (1988). In the past few years, CFDLs have been discovered in laboratory experiments in which a low-pressure plasma is forced to expand from a high magnetic field source region to a low magnetic field diffusion region. A strong CFDL or electrostatic shock with $e \Phi / k_{\mathrm{B}} T_{e} \approx 3$ ( $\Phi$ is the electric potential, $e$ the electron charge, $k_{\mathrm{B}}$ Boltzmann's constant, and $T_{e}$ the electron temperature) and a thickness of less than 50 Debye lengths was experimentally obtained by Charles \& Boswell (2003) in an expanding, high-density helicon-sustained radio frequency (13.56 MHz) discharge. The spontaneous formation of this CFDL was subsequently confirmed in other experimental systems (Sun et al. 2005; Sutherland et al. 2005) and successfully modeled using particle-in-cell simulations (Meige et al. 2005).

In this experiment the rapid potential decrease is associated with the maximum gradient of the magnetic field, the potential is self-consistently generated by the plasma itself, and there is no current flowing through an external circuit. The plasma electrons are heated by the radio-frequency field in the source, provide the power to maintain the DL, and hence accelerate the ions created in the source out into the diffusion chamber. A supersonic beam of hydrogen (with a sonic Mach number of 2) on the low potential side was discovered by Charles (2004) 


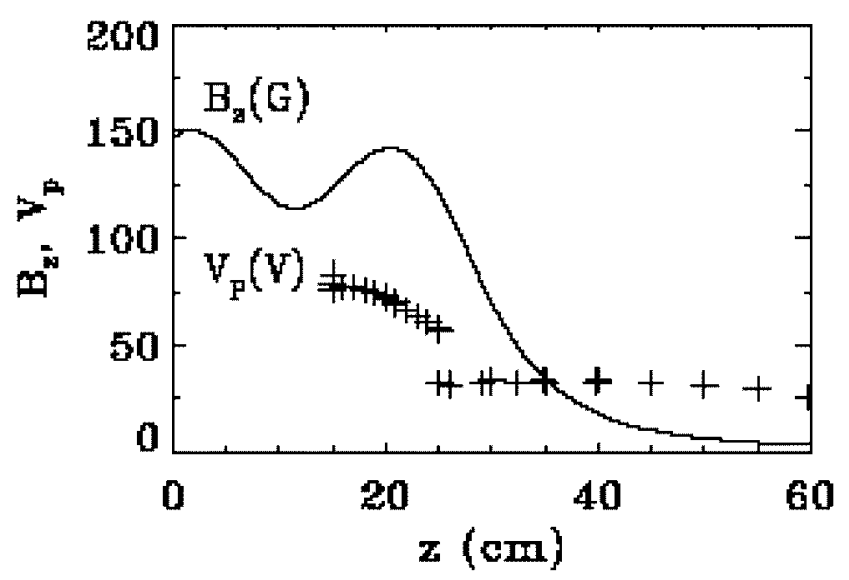

Fig. 1.- Magnetic field strength (in gauss) in the electric DL set up in a laboratory device. The plasma potential (in volts on the same scale) is indicated by plus signs.

and described by Charles \& Boswell (2004). The CFDL is shown in Figure 1 by the strong potential drop at $z=25 \mathrm{~cm}$. Particle acceleration in the potential drop was detected downstream of the CFDL, with typical measured ion velocities of $10 \mathrm{~km} \mathrm{~s}^{-1}$.

Present thinking indicates that the pressure gradient created by the change in the plasma density gives rise to a potential gradient that can be thought of as retarding the plasma electrons but accelerating the ions. If the acceleration in this field brings the ions to supersonic velocities, an instability develops, leading to the DL. This can only occur in sufficiently collisionless plasmas where the mean free path for momentum exchange (or charge exchange) for the ions is greater than the scale length of the potential gradient.

This plasma expansion, and the concomitant formation of an electric DL, offers applications in solar physics, in particular when one considers the supply of plasma to the corona and the acceleration of the nascent solar wind in the solar transition region (for an introduction to its physics, see Mariska 1992). A comparison of typical plasma parameters in a coronal funnel and electric DL in the laboratory is provided in Table 1. The parameters in the four top rows are taken from measurements or derived from observations, whereas the ones in the three subsequent rows are theoretically calculated and rounded up. The electron gyroradius $R_{e^{-}}$is based on the most probable thermal speed, and the proton gyroradius $R_{\mathrm{H}^{+}}$on the quoted ion drift speed $V_{i}$.

The four top lines of Table 1 (where $n_{e}$ is the electron density) indicate that there is a striking similarity of a number of important parameters between the Sun and the laboratory; however, the physical dimensions are dramatically different, with the diameter $(L)$ of the laboratory experiment being around 7 orders of magnitude less than the coronal funnel. Correspondingly, the time $\left(t=L / V_{i}\right)$ it takes an ion to transit the plasma vessel vastly differs from the one to pass a funnel. So, where the gyroradius of a proton with a speed of $10 \mathrm{~km} \mathrm{~s}^{-1}$ would in the laboratory be about the size of the diffusion chamber, in a coronal funnel it would be 6 orders of magnitude smaller (Table 1). This is similarly the case for the mean free paths for ion-ion collisions, which would be relatively much smaller than a coronal funnel and much larger than the laboratory. Interestingly, this has been implicitly assumed for the results of $\mathrm{Tu}$ et al. (2005), who assume that the measured velocities of the $\mathrm{Ne}^{7+}$ reflects the bulk velocity of the plasma streaming out of the coronal funnel.

The plasma density decreases proportionally to the magnetic field, and the fields in the coronal funnel and the plasma vessel are of the same order of magnitude. The plasma density decreases in both cases, and the observed plasma acceleration, which is directly related to the strength of the DL, is almost the same, as inspection of Table 1 shows. The strength of the DL is not strongly dependent on the amplitude of the magnetic field, although a minimum value of a few tens of gauss is necessary for the DL to form in the experimental system. The strength of the DL mainly depends on the electron temperature $\left(e \Phi / k_{\mathrm{B}} T_{e}=3\right)$, and the corresponding electric field of the DL is typically in the $250-500 \mathrm{~V} \mathrm{~m}^{-1}$ range. The DL strength obtained in self-consistent particle-in-cell simulations and in other experimental devices with slightly different magnetic field divergence is found to be between 2 and 4 times the electron temperature (Meige et al. 2005; Sun et al. 2005), in good agreement with our laboratory experiment (Charles \& Boswell 2003).

Estimates of the Debye length $\lambda_{\mathrm{D}}$ and of the electron and proton gyroradius (see again Table 1) also indicate similar values in the laboratory experiment and in the coronal funnel, although the physical dimensions of the two systems are radically different. In addition, a temporal study of the laboratory experiment has shown that the DL is created during plasma breakdown at about $100 \mu \mathrm{s}$ after the radio-frequency power is applied and is stable thereafter (Charles 2005a).

There is one property that needs considerable thought, and that is the stability of a DL having an area of nearly a million square kilometers! Nevertheless, physics is based on scalability, and since auroral DLs exist on a scale much greater than the laboratory, we postulate, with some trepidation, that they can exist near the surface of the Sun.

\section{CORONAL FUNNELS}

As the interplanetary fast solar wind travels along magnetic field lines that originate in the solar photosphere below coronal

TABLE 1

Characteristic Plasma Parameters of Electric Double Layers AND CORONAL FUNNELS

\begin{tabular}{ccccc}
\hline \hline Variable & DL Source & Funnel Bottom & DL Diffusion Chamber & Funnel Top \\
\hline$n_{e}\left(\mathrm{~cm}^{3}\right) \ldots \ldots \ldots$ & $10^{10}$ & $10^{10}$ & $10^{9}$ & $10^{8}$ \\
$T_{e}(\mathrm{~K}) \ldots \ldots \ldots \ldots$ & $10^{5}$ & $10^{4}$ & $10^{5}$ & $10^{5}$ \\
$B(\mathrm{G}) \ldots \ldots \ldots \ldots$ & 130 & 50 & 10 & 5 \\
$V_{i}\left(\mathrm{~km} \mathrm{~s}^{-1}\right) \ldots \ldots$ & 2 & 1 & 10 & 10 \\
$\lambda_{\mathrm{D}}(\mathrm{mm}) \ldots \ldots \ldots$ & 0.2 & 0.07 & 0.7 & 2 \\
$R_{e^{-}}(\mathrm{cm}) \ldots \ldots \ldots$ & 0.08 & 0.06 & 1 & 2 \\
$R_{\mathrm{H}^{+}}(\mathrm{cm}) \ldots \ldots \ldots$ & 0.15 & 0.2 & 0.3 & 20 \\
$L(\mathrm{~m}) \ldots \ldots \ldots \ldots$ & 0.3 & $2 \times 10^{6}$ & $3 \times 10^{-5}$ & $1.5 \times 10^{6}$ \\
$t(\mathrm{~s}) \ldots \ldots \ldots \ldots$ & $1.5 \times 10^{-4}$ & $2 \times 10^{3}$ & & 1.5 \\
\hline
\end{tabular}




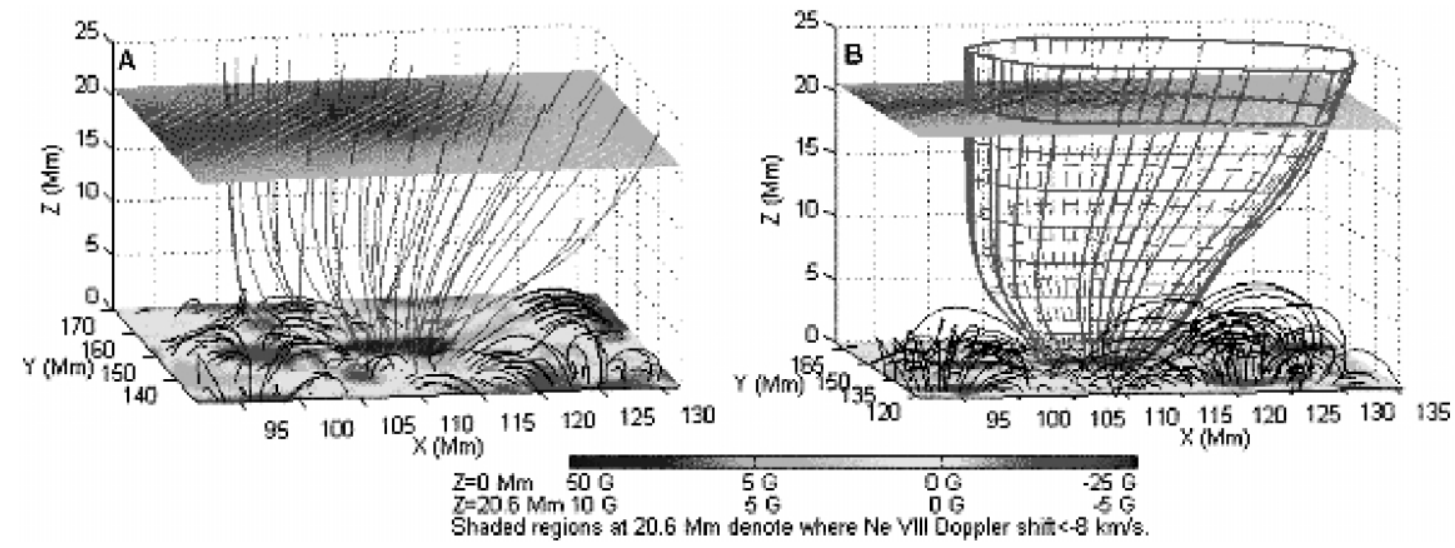

FIG. 2.-Magnetic funnel in the solar corona. (a) Correlation of open fields with $\mathrm{Ne}^{7+}$ outflow (dark hatched shading indicates velocities greater than 8 km $\mathrm{s}^{-1}$ ). The magnetic field is indicated in color (coding at bottom). (b) Illustration of the funnel and unipolar flux constriction by the surrounding small bipolar loops (after Tu et al. 2005). [See the electronic edition of the Journal for a color version of this figure.]

holes (regions of open field lines), these must be the source of this rapid flow. The specific regions were identified by Hassler et al. (1999), Xia et al. (2003), and Tu et al. (2005) as the coronal funnels anchored in the magnetic network lanes. It is now recognized (Marsch et al. 2003) that high-frequency waves may be relevant, or that the actual acceleration mechanism even remains hidden, but apparently it must be located closer to the solar surface than previously thought. To achieve a rapid nearSun acceleration it is necessary to find low in the funnels a mechanism that can accelerate the plasma along open fields to supersonic velocity (i.e., locally to a few tens of kilometers per second within several megameters). This is the problem we wish to investigate using the mechanism of the CFDL in a coronal funnel to provide basic acceleration.

The detailed magnetic structure of the solar wind source region was recently elucidated by Tu et al. (2005), who established the source region location of the solar wind in coronal funnels by a correlation analysis between the Doppler-shift and radiance maps of vacuum ultraviolet emission lines with charts of the magnetic field as obtained at transition region heights by a force-free field extrapolation from photospheric magnetograms. Specifically, the heavy $\mathrm{Ne}^{7+}$ ions (forming at about $0.6 \mathrm{MK}$ ) used as tracers of the bulk flow were found to radiate mostly around $20 \mathrm{Mm}$, where they have outflow speeds of about $10 \mathrm{~km} \mathrm{~s}^{-1}$, whereas the $\mathrm{C}^{3+}$ ions (forming at $0.1 \mathrm{MK}$ ) were observed to radiate mainly around $5 \mathrm{Mm}$ and had no average flow speed. Thus was the height range of the fast solar wind source region determined.

An example of a funnel is shown in Figure 2 after Tu et al. (2005). The magnetic field magnitude $B$ typically changes from $50 \mathrm{G}$ in the chromosphere at $1 \mathrm{Mm}$ to $5 \mathrm{G}$ at $20 \mathrm{Mm}$ above the photosphere where the plasma beta is low. It should be emphasized that the field was not measured but obtained by force-free-field extrapolation from a measured photospheric magnetogram. Below $5 \mathrm{Mm}$ near the funnel neck the plasma has a higher beta, may undergo reconnection, and may still be governed by the convection of the chromospheric network. Here the furnace model of Axford \& McKenzie (1997) is invoked, which assumes that reconnection is playing a major role in releasing plasma and freeing magnetic energy. Tu et al. (2005) suggested that the initial plasma heating is achieved in the small loops adjacent to the funnel.

The heating of the corona and the acceleration of the solar wind remain matters of intensive research and debate; for recent reviews see Axford \& McKenzie (1997) and Marsch et al. (2003). Considering that coronal heating and solar wind ac- celeration are closely related processes, the models of the solar wind were extended throughout the solar transition region down to the top of the chromosphere (McKenzie et al. 1998), to include the region where hydrogen becomes fully ionized. Also, wave-driven fluid models were developed, with the assumption that the solar wind is created in the coronal funnels (Marsch \& Tu 1997; Hackenberg et al. 2000), in which the nascent solar wind flow is pushed up by the gradients of the intrinsic thermal pressure and external wave pressure. The strength of a laboratory DL with a potential drop of $25-50 \mathrm{~V}$ (as explained in the previous section) corresponds to about a hundredth of the total energy required to generate the solar wind (Marsch et al. 2003), which is of the order of a few keV. Thus the proposed DL in a coronal funnel can provide the initial plasma heating and acceleration in the transition region, but it may not be strong enough to give the solar wind its terminal speed.

\section{PROPERTIES OF DOUBLE LAYERS IN LABORATORY PLASMA}

The recent terrestrial experiments on CFDLs have all been carried out at low pressures (around 1 mtorr) using a radiofrequency plasma source placed near the maximum of a diverging magnetic field. The DL appears to form in the region of expanding magnetic field where the gradient is greatest. The strength of this CFDL is of the order of a few $k_{\mathrm{B}} T_{e}$ and hence is classified as small. There is no definitive theory yet about the CFDL, yet a number of properties emerged from the experimental work. It is the change in the plasma pressure that generates an electric potential which is given quite accurately by Boltzmann's equation, assuming massless electrons having a constant temperature.

The CFDL appears to depend on the source plasma being able to float electrically and hence being isolated from the conducting walls of the diffusion plasma, at Earth potential in this case. The power necessary to maintain the CFDL comes from the high-energy source electrons that charge it up. The potential drop accelerates the ions into a narrow beam having a divergence of around $5^{\circ}$. Gasses ranging from hydrogen to xenon have been successfully used, so it would appear that the CFDL is dominated by the electron dynamics. Computer modeling of the two-dimensional trajectories of the ions accelerated by the CFDL into the diverging magnetic field are in good agreement with the experiment (Gesto et al. 2006).

The experiment has demonstrated the existence of DLs with hydrogen, argon, xenon, and oxygen, and so it can be reasonably assumed that it will occur independently of the mass of 
the ion (Charles 2005b). Although the paper by Tu et al. (2005) talks about the measurement of multiply charged ions such as $\mathrm{Ne}^{7+}$, it should be remembered that these ions are very much in the minority, the most abundant coronal ions being protons and alpha particles, which cannot be detected spectroscopically.

When operating with argon gas, an ion velocity of $2 \mathrm{~km} \mathrm{~s}^{-1}$ was measured upstream of the DL and a velocity of $10 \mathrm{~km} \mathrm{~s}^{-1}$ was measured downstream of the DL. The electron temperature was about $10^{5} \mathrm{~K}$. These results are consistent with the measurements reported by Tu et al. (2005), where $\mathrm{Ne}^{7+}$ ions are observed to have a velocity of about $10 \mathrm{~km} \mathrm{~s}^{-1}$ at $20 \mathrm{Mm}$ in a region where the electron temperature is $10^{5} \mathrm{~K}$. The limiting mean free path in the laboratory experiment is charge exchange of ions on neutrals, whereas this is clearly not the case in coronal funnels, and hence we would not expect an ion beam produced by a DL to be degraded in the corona in the same way it is in the laboratory.

\section{THE DOUBLE LAYER IN A CORONAL FUNNEL}

Present models of the origin of the fast solar wind invoke reconnection, the furnace model, and wave heating in small side loops. Although these possibilities are not unreasonable, we prefer to rest our suggestion on solid evidence from laboratory experiments. The solar DL has the advantage that the ions are directly accelerated in a fairly clear (if poorly understood) way, and the trajectory calculations strongly suggest that the beam thus created does not expand with the magnetic field but streams directly outward from the surface of the Sun, assuming the magnetic expansion axis to be normal to the solar surface. In addition, the ion beam will be streaming through a background plasma that very probably will lead to instabilities of the two-stream type. These instabilities will tend to move energy from the directed beam to the thermal distribution, thereby collisionlessly heating the ions.

A simple estimate of the dissipation by the CFDL can be obtained by assuming all the ions (predominantly protons with a density of $10^{10} \mathrm{~cm}^{-3}$ ) at the bottom of the coronal funnel and across a diameter of around $1000 \mathrm{~km}$ are accelerated from rest to $10 \mathrm{~km} \mathrm{~s}^{-1}$. This yields a power of greater than $10^{14} \mathrm{~W}$, which must be furnished by electrons in the lower regions of the coronal funnels.

\section{CONCLUSION}

The present paper does not call on the traditional explanations of solar wind plasma supply and initial acceleration; rather, we would like to suggest that the basic mechanism lying at the origin of the solar wind is ion acceleration through an electrostatic double layer created by chromospheric plasma expanding upward in a coronal funnel. The similarity of the laboratory experiments with the solar coronal funnels is striking. For both coronal funnels and the plasma vessel, the plasma density decreases proportionally to the magnetic field, the magnetic fields are of the same order of magnitude, and the plasma acceleration is almost the same. In the laboratory experiments, the electrons heated by the applied radio frequency in the source supply the power for the double layer, whereas in case of the solar atmosphere an electron energization mechanism still has to be identified at the bottom of a funnel. Nevertheless, we believe that a double layer is a way to accelerate the plasma up into the corona.

\section{REFERENCES}

Axford, W. I., \& McKenzie, J. F. 1997, in Cosmic Winds and the Heliosphere, ed. J. R. Jokipii, C. P. Sonett, \& M. S. Giampapa (Tucson: Univ. Arizona Press), 31

Block, L. P. 1978, Ap\&SS, 55, 59

Charles, C. 2004, Appl. Phys. Lett., 84, 332 2005a, Phys. Plasmas, 12, 044508

. 2005b, Helicon Double Layer Thrusters, in Proc. 27th Int. Conf. on Phenomena in Ionized Gases (Eindhoven: ICPIG), http://www.icpig2005.n1/ cd/D:/Proceedings_ICPIG_2005.html

Charles, C., \& Boswell, R. W. 2003, Appl. Phys. Lett., 82, 1356 2004, Phys. Plasmas, 11, 1706

Ergun, R. E., et al. 2001, Phys. Rev. Lett., 87, 045003

Gesto, F. N., Blackwell, B. D., Charles, C., \& Boswell, R. W. 2006, J. Propulsion \& Power, 22, 24

Goertz, C. K., \& Boswell, R. W. 1979, J. Geophys. Res., 84, 7239

Hackenberg, P., Marsch, E., \& Mann, G. 2000, A\&A, 360, 1139

Hairapetian, G., \& Stenzel, R. L. 1988, Phys. Rev. Lett., 61, 1607

Hassler, D. M., Dammasch, I. E., Lemaire, P., Brekke, P., Curdt, W., Mason, H. E., Vial, J. C., \& Wilhelm, K. 1999, Science, 283, 810

Jacobsen, C., \& Carlqvist, P. 1964, Icarus, 3, 270
Mariska, J. T. 1992, The Solar Transition Region (Cambridge: Cambridge Univ. Press)

Marsch, E., Axford, W. I., \& McKenzie, J. M. 2003, in Dynamic Sun, ed. B. N. Dwivedi (Cambridge: Cambridge Univ. Press), 374

Marsch, E., \& Tu, C.-Y. 1997, Sol. Phys., 176, 87

McKenzie, J. F., Sukhorukova, G. V., \& Axford, W. I. 1998, A\&A, 330, 1145

Meige, A., Boswell, R. W., Charles, C., \& Turner, M. M. 2005, Phys. Plasmas, 12,052317

Perkins, F. W., \& Sun, Y. C. 1981, Phys. Rev. Lett., 46, 115

Raadu, M. A. 1989, Phys. Rep., 178, 25

Stenzel, R. L., Gekelman, W., \& Wild, N. 1983, J. Geophys. Res., 88, 4793

Sun, X., Keese, A. M., Biliou, C., Scime, E. E., Meige, A., Charles, C., \& Boswell, R. W. 2005, Phys. Rev. Lett., 95, 025004

Sutherland, O., Charles, C., Plihon, N., \& Boswell, R. W. 2005, Phys. Rev. Lett., 95, 205002

Tu, C.-Y., Zhou, C., Marsch, E., Xia, L.-D., Zhao, L., Wang, J.-X., \& Wilhelm, K. 2005, Science, 308, 519

Volwerk, M., \& Kuijpers, J. 1994, ApJS, 90, 589

Xia, L. D., Marsch, E., \& Curdt, W. 2003, A\&A, 399, L5 